\title{
Percepción de conductores de transporte urbano, sobre calidad de vida laboral
}

\author{
Perception on quality of life at work from drivers of urban transport
}

Nancy Viviana Oviedo-Oviedo'*, José Manuel Sacanambuy-Cabrera²

Sonia Maritza Matabanchoy-Tulcan³, Christian Alexander Zambrano-Guerrero4

1 Psicóloga. Egresada Universidad de Nariño. Pasto, Colombia. e-mail: vivis.2ovi@gmail.com

2 Psicólogo. Egresado Universidad de Nariño. Pasto, Colombia. e-mail: josemanuelsacanambuy@gmail.com

3 Psicóloga, Candidata a Doctora en Psicología. Magíster en Gerencia del Talento Humano. Docente tiempo completo e Integrante del grupo de investigación COGNICED del Programa de Psicología, Universidad de Nariño. Consultora de Talento Humano. Pasto, Colombia. e-mail: somapsicologa@yahoo.es

4 Psicólogo, Magíster en Educación desde la Diversidad. Docente del Programa de Psicología, Universidad de Nariño. Integrante del grupo de investigación Psicología y Salud e integrante del grupo de investigación COGNICED del Programa de Psicología, Universidad de Nariño. Pasto, Colombia. e-mail: christianzambris@hotmail.com

Oviedo-Oviedo NV, Sacanambuy-Cabrera JM, Matabanchoy-Tulcan SM, Zambrano-Guerrero CA. Percepción de conductores de transporte urbano, sobre calidad de vida laboral. Univ. Salud. 2016;18(3):432-446. DoI: http://dx.doi.org/10.22267/rus.161803.49

\begin{abstract}
Resumen
Introducción: La calidad de vida laboral en las organizaciones es un aspecto transversal en la gestión del talento humano ya que esta área además de implementar, mantener y mejorar los sistemas de gestión de calidad y los procesos de prestación del servicio, centra su intervención en el componente humano. Objetivo: Caracterizar la calidad de vida laboral a través de los procesos de gestión del talento humano del Sistema Estratégico de Transporte Público de la ciudad de Pasto. Materiales y métodos: Se empleó un paradigma cuantitativo de tipo descriptivo, con el fin de identificar, describir y analizar la calidad de vida laboral por medio de la salud, bienestar y percepción de trabajo, contando con la participación de 468 conductores. Se utilizó técnicas de recolección de información como la revisión bibliográfica, una escala de actitudes tipo Likert y una encuesta de autoreporte de morbilidad sentida, los resultados de estas aplicaciones fueron sistematizados por medio del programa estadístico IBM SPSS Statistics. Resultados: Dentro de esta población existe una actitud favorable hacia sus condiciones de salud (79,3\%), sin embargo, estos autoreportes muestran la presencia de dolores físicos que afectan la salud de los trabajadores (dolores de espalda, cintura y manos), una actitud muy favorable frente al bienestar laboral $(77,6 \%)$ y una actitud muy favorable hacia la percepción del trabajo $(75,2 \%)$. Conclusiones: Los resultados permiten identificar las áreas por fortalecer, promover la calidad de vida de los conductores (salud laboral, bienestar laboral y percepción de trabajo), mejorar la prestación del servicio y favorecer el desarrollo de la ciudad, resaltando la labor del psicólogo organizacional dentro de la empresa.
\end{abstract}

Palabras clave: Salud laboral; trabajadores; riesgos laborales. (Fuente: DeCS, Bireme).

\begin{abstract}
Introduction: Quality of working life in organizations is a transversal aspect in the management of human talent, since this area not only implements, maintains and improves quality management systems and processes of service
\end{abstract}


provision, but also focus its intervention on the human component. Objective: Characterize the quality of working life through the processes of human talent management of the Strategic System of Public Transport in the city of Pasto. Materials and methods: Therefore, a quantitative paradigm of a descriptive type was used in order to identify, describe and analyze the quality of work life through health, well-being and perception of work, with the participation of 468 drivers. The methods used for data collection were bibliographic review, a Likert-type attitude scale and a self-report questionnaire for morbidity. The results of these applications were systematized using the statistical software IBM SPSS Statistics. Results: Within this population there is a favorable attitude towards their health conditions (79.3\%); however, these self-reports show the presence of physical pains that affect the health of workers (back pains, waist and hands), a very favorable attitude when facing the labor welfare (77.6\%) and a very favorable attitude towards the perception of work (75.2\%). Conclusions: The results allow to identify the areas to be strengthened: promoting the drivers' quality of life (occupational health, labor well-being and job perception), improving provision of service and promoting the development of the city where the labor of the organizational psychologist is highlighted within the company.

Keywords: Occupational health; workers; occupational hazards. (Source: DeCS, Bireme).

\section{Introducción}

Los sistemas de transporte constituyen una pieza clave en el funcionamiento de las ciudades modernas, debido a que ofrecen un servicio que atiende a la mayoría de la población ${ }^{1}$; este facilita el transporte de personas a diversos lugares para desempeñar sus labores cotidianas, e influyen de esta manera en la dinámica social y económica ${ }^{2}$, por ello, es preciso el estudio, la innovación, el mejoramiento y la adecuación de los sistemas de transporte que respondan a las necesidades de la población y de sus conductores.

Entre las necesidades predominantes en la prestación del servicio de transporte público, se encuentran la reducción de la accidentalidad, el congestionamiento vial, el consumo de energía, la contaminación, entre otros ${ }^{3}$. La accidentalidad es una consecuencia negativa de los medios de transporte, ocasionada por factores asociados al estado, tipo de vías y vehículos, y en mayor medida a factores asociados a los conductores ${ }^{4}$ con un $71 \%$ al $93 \%$, según informa la Administración de la Seguridad del Tráfico en Autopistas (EE.UU)5. La accidentalidad, según reportes médicos, es causada por el cansancio y la somnolencia de conductores fatigados, que disminuyen los niveles de atención, concentración y pérdida de respuesta inmediata a situaciones que lo exigen 6 .
El Ministerio de Transporte de Colombia hace una evaluación en cuanto al nivel de accidentalidad desde el año 2002 al 2014 y encuentra que los índices de muerte por accidentalidad han sido ascendentes en el nivel nacional; en el año 2014 se registró 180.519 accidentes, con 6.352 casos de muerte, a diferencia de 2004 donde se presentaron mayor número de accidentes (229.184), pero menor número de muertos (5.483). En el análisis por departamento se describe que en el 2014 Antioquia, Valle del Cauca y Bogotá presentaron mayores casos de muerte por accidentes con 986, 775 y 640 casos respectivamente; en el departamento de Nariño las muertes por accidentalidad fueron de 175, cifra elevada teniendo en cuenta que la población en el departamento es menor ${ }^{7}$.

Con relación a lo anterior, son los conductores las personas con mayor riesgo de presentar un accidente de tránsito, esto se debe al tiempo de exposición diaria a los factores de riesgo asociados a la conducción, de aquí la importancia, de tomar los accidentes de tránsito como accidentes laborales para esta población, porque cualquier hecho ocurrido con causa $\mathrm{u}$ ocasión laboral según la Ley 1562 de 2012 es un accidente laboral ${ }^{8} \mathrm{y}$, por ende, considerado como un riesgo laboral. 
En congruencia con la Ley, el Ministerio de Trabajo de Colombia ${ }^{9}$, a través de las Administradoras de Riesgos Laborales, presentaron el reporte de accidentalidad correspondiente al año 2012, donde se registraron 609.881 accidentes laborales en el nivel nacional; e indica que el sector transporte, cuenta con un número elevado de enfermedades laborales con 30.635 casos reportados. FASECOLDA (Federación de Aseguradores Colombianos) en el 2013 presentó reportes que indican que el nivel de accidentalidad laboral en conductores es del 5,74\% y de enfermedades laborales del $54,12 \%{ }^{10}$, estas cifras reflejan que además de trabajar con la accidentalidad laboral es importante abordar las enfermedades laborales, diferenciando las causas que las ocasionan.

Algunas de las causas de accidentalidad laboral y enfermedad laboral pueden ser explicadas a través de la adopción de posturas inadecuadas, los estilos de vida no saludables (el consumo de alcohol, tabaquismo, falta de actividad física, la dieta poco saludable), y de razón psicológica (alta tensión debido a las condiciones peligrosas del trabajo, hábitos irregulares de sueño y el estrés) ${ }^{11}$.

Naranjo describe, que el estrés implica cualquier factor externo o interno que induce a un aumento en el esfuerzo de la persona para mantener un estado de equilibrio dentro de sí misma y en relación con su ambiente ${ }^{12}$, "el estrés puede ser positivo (eustrés) o negativo (distrés) según los recursos que posea cada persona para dar respuesta a situaciones que provocan tensión ${ }^{13}$."

Córdoba y Moreno ${ }^{14}$, por su parte, resaltan la necesidad de investigar sobre los desencadenantes de estrés, ya que este influye en la forma de conducir, tiene efectos negativos en el conductor y es causante de accidentes. Entre algunos de los elementos que desencadenan el estrés son: el trabajo en solitario, la falta de participación, las horas de trabajo irregulares, la violencia, la agresión y la presión. De esta forma, los trabajadores del sector transporte terrestre están más afectados por el estrés laboral que otros trabajadores con un 33.3\% en comparación con un $23.7 \% 15$. En lo que corresponde a posturas inadecuadas, específicamente en la posición sedente, se encuentran diversos factores que intervienen en las manifestaciones de dolor en las regiones dorso lumbares, las cuales se deben generalmente a una posición incorrecta al sentarse, la falta de apoyo lumbar y la falta de apoyo en antebrazos, entre otros, que pueden predisponer al individuo a niveles más altos de malestar general, tales como dolor, pesadez o sensación de hormigueo en diferentes partes del cuerpo ${ }^{16}$.

En esta medida, Farmer afirma que son pocos los conductores conscientes de que cada año miles de personas sufren lesiones de espalda y cuello como resultado de la forma en que se sientan $u$ operan sus vehículos, considerando que un factor susceptible de ser estudiado es la ergonomía del conductor, la cual se centra en los aspectos sanitarios de la conducción, basándose en la biología, la psicología, la ingeniería y el diseño para crear entornos de vehículos que se adapten a las personas ${ }^{17}$.

Otro factor que incide sobre la salud del conductor es el sedentarismo, que se relaciona con enfermedades cardiovasculares, obesidad, diabetes $^{11}$ y debido a su estilo de vida $y$ exposición ocupacional ${ }^{18}$ es imprescindible evaluar dietas y motivar la realización de actividad física.

Teniendo en cuenta los factores expuestos hasta el momento, se podría inferir que existe una diversidad de causas que ocasionan la pérdida del equilibrio en el estado de salud de un conductor de transporte urbano; dichos factores, son contemplados bajo la luz de la teoría de la multicausalidad propuesta por la Organización Mundial de la Salud (OMS), en donde la salud y enfermedad de las personas se concibe como el resultado de múltiples factores interactuantes que inciden significativamente en el proceso que se da entre la vida y muerte de cada individuo en una sociedad determinada ${ }^{19}$.

Bajo esta teoría se debe contemplar inicialmente la definición de salud, comprendiéndola, como 
un estado de completo bienestar físico, mental y social, y no solamente la ausencia de afecciones o enfermedades ${ }^{19}$.

Retomando la teoría ya expuesta, en torno a las causas que inciden sobre salud de los conductores, se podría plantear, que un gran porcentaje de los conductores de transporte público urbano son afectados a nivel físico, mental y social por aquellos factores inherentes a su profesión, y estos a su vez son considerados como riesgos laborales, provocando en muchas ocasiones ausentismo laboral, éste es un fenómeno costoso, que implica un impacto financiero para la empresa, para los trabajadores, las instituciones de seguridad $\operatorname{social}^{20} \mathrm{y}$, sin lugar a dudas, implica una afectación en la calidad de vida de la persona, dado que un problema de salud incide en la todas las dimensiones del trabajador.

La calidad de vida (sensación de bienestar físico, psicológico y social), es entendida como el estado de satisfacción, derivado de la realización de las potencialidades de la persona, la cual posee aspectos subjetivos y objetivos. Dentro de los aspectos subjetivos incluye la expresión emocional, la productividad personal, la seguridad y salud percibidas. Como aspectos objetivos, se incluye el bienestar material, las relaciones armónicas con el ambiente físico y social, y la salud objetiva ${ }^{21}$. Esta calidad de vida, al ser transversal a todas las prácticas de gestión de talento humano, requiere actividades direccionadas hacia el mejoramiento de la salud laboral, bienestar laboral y percepción del trabajo, categorías que abordan en forma global los aspectos subjetivos de la calidad de vida.

La salud laboral hace alusión a la promoción de la salud en el lugar de trabajo, vigilar las condiciones de trabajo, además de la identificación, eliminación o modificación de factores que sean perjudiciales para la salud de los colaboradores ${ }^{22}$. El bienestar laboral se relaciona con características específicas del trabajo (variedad de tarea, autonomía, posibilidad de utilizar conocimientos), prácticas de alto rendimiento en el trabajo (trabajo en equipo y comunicación horizontal y vertical) y factores que afectan el entorno del trabajo (relaciones con la gerencia, seguridad en el empleo y entorno social) ${ }^{23}$.

La salud y el bienestar son valorados por el trabajador a través de la percepción del trabajo (proceso donde el trabajador extrae información del ambiente de trabajo, donde se reconoce y se aprecia el mundo exterior mediante los estímulos recibidos por los sentidos y las respuestas motoras provocadas por esos estímulos $)^{24}$. Su importancia reside, en el hecho de que el comportamiento no es una resultante de las condiciones de trabajo existentes, sino que depende de las percepciones que tenga el colaborador de estos factores, se relaciona por tanto, con la interacción entre características personales y organizacionales ${ }^{25}$.

Por esta razón, surgió la iniciativa de recolectar información a través de las percepciones y actitudes de los conductores de bus urbano con respecto a las categorías de salud laboral, bienestar y percepción del trabajo, con el objetivo de identificar, describir y analizar la calidad de vida laboral, el cumplimiento de estos objetivos permitió inicialmente la identificación de necesidades que deben ser tenidas en cuenta para intervenciones oportunas.

Ahora bien, el identificar aquella necesidad brinda las directrices del trabajo del psicólogo organizacional, basándose en la investigación de factores que afectan a los trabajadores; en esta medida facilita el diseño de programas que contribuyen al mejoramiento de la seguridad y la salud laboral 26 .

\section{Materiales y métodos}

Se realizó un estudio cuantitativo de carácter descriptivo que procuró estudiar los fenómenos de interés de la investigación de la psicología organizacional, para este caso la calidad de vida laboral que es inherente a la gestión de talento humano, por medio de mediciones objetivas y análisis que involucran métodos numéricos y cuantitativos ${ }^{27}$, se utilizó técnicas de recolección de información como la revisión bibliográfica, 
una escala de actitudes tipo Likert como escala de respuestas de percepciones y actitudes, y una encuesta de autorreporte de morbilidad sentida $^{28}$.

La revisión bibliográfica consistió en la búsqueda de investigaciones realizadas en torno a gestión del talento humano, sistemas de transporte, normatividad en cuanto a seguridad y salud en el trabajo, prácticas de talento humano, medición y evaluación psicológica en bases de datos (SciELO, Redalyc, EBSCO ), revistas científicas indexadas y libros virtuales.

Para la recolección de información se construyó en el equipo de trabajo una escala de actitudes tipo Likert, que fue valorada por 5 jueces expertos dentro de los campos de la medición y evaluación psicológica, psicología organizacional y salud ocupacional, para ellos se diseñó un formato con los ítems a evaluar y tres casillas con criterios como pertinencia, claridad y redundancia, posterior a ello, se realizó los ajuste sugeridos por los jueces expertos dando como resultado la escala de actitudes que fue sometida a prueba piloto y finalmente aplicada a la población objeto de estudio, esta escala midió las actitudes frente a la calidad de vida laboral desde el punto de vista de la GTH, el Alfa de Cronbach general fue de 0,860 y los Alfa de Cronbach por categoría fueron: salud laboral 0,758 , bienestar 0,853 y percepción del trabajo 0,732 esta escala permitió identificar dimensiones de la calidad de vida laboral como la salud laboral (conformada por cuatro subcategorías condiciones de salud, condiciones de trabajo, estilos de vida y tiempo libre), bienestar laboral (posee tres subcategorías bienestar laboral, familia y clima organizacional) y percepción laboral (subcategorías que son satisfacción laboral y atención al usuario). La categorización de cada uno de los ítems correspondió a las opciones de respuesta siempre, casi siempre, algunas veces, casi nunca y nunca.

Para contrastar los resultados en la categoría de salud laboral, se aplicó una encuesta de autorreporte de morbilidad sentida (Kuder Richardson de 0,8851) suministrada por la
Administradora de Riesgos Laborales (ARL) a la cual se encuentran afiliados los trabajadores, que evalúa antecedentes médicos y manifestaciones actuales, este presenta una forma de respuesta dicotómica (sí/no), de los cuales se retomó los resultados más significativos relacionados con las condiciones de salud de los conductores. Esta información se sistematizó y analizó utilizando el programa estadístico IBM SPSS Statistics versión 20, que permitió obtener puntuaciones, agrupaciones y porcentajes de los resultados para su posterior análisis y discusión.

La población estuvo constituida por 538 conductores del Sistema Estratégico de Transporte Público de Pasto (SETP), de los cuales se obtuvo una muestra de 468 conductores en edades comprendidas entre los 20 y 73 años, a través un muestreo aleatorio simple, con un nivel de confianza del $98 \%$ y un margen de error del 2\%. El procedimiento de aplicación inició con la firma del consentimiento informado aceptando participar en el estudio y aclarando la confidencialidad en el uso de la información posteriormente se procedió a la aplicación de los instrumentos.

\section{Consideraciones éticas}

La investigación se realizó bajo la normatividad de la Ley 1090 de $2006^{29}$, donde se establece el Código Deontológico y Bioético del psicólogo, el cual establece que el manejo de información es estrictamente confidencial, por tanto el análisis y publicación de este artículo se realiza de forma general con el fin de proteger la identidad de los participantes, se cuenta además con la previa autorización de los conductores a través de la firma de un consentimiento informado y con el aval de la empresa donde se recolectó la información.

\section{Resultados}

Los datos sociodemográficos indican que el $100 \%$ de los conductores de la muestra son hombres, de los cuales la mayor parte ha laborado dentro de la empresa entre 1 año o menos $(23,2 \%)$ y de 1 a 5 años $(24,1 \%$ ) a diferencia de los conductores que han permanecido más tiempo en sus cargos, estos se 
hallan entre 30 a 35 años $(1,3 \%)$ y de 35 a 40 años $(0,4 \%)$, en cuanto a la jornada laboral esta se encuentra en un promedio de 15 horas.

Las edades más frecuentes de los conductores oscilan entre los 20 a 30 años $(23,9 \%)$ y de los 30 a 40 años (36,1\%), y en menor proporción se encuentra entre las edades de 50 a 60 años $(15,8)$ y 60 en adelante $(1,3 \%)$, en este sentido el rango de edad promedio va desde los 20 a los 50 años de edad. En cuanto al peso más frecuente en la población de los conductores es de los 70 a 80 kilogramos $(34,2 \%)$ y de 80 a 90 kilogramos $(31,6 \%)$, entre los 100 a 110 kilogramos $(5,3 \%)$ y 110 a 120 kilogramos (0,4\%). El 1,3\% de los conductores reporta no haber recibido ningún grado de escolaridad, el $44,7 \%$ realizó sus estudios de nivel primario, el 50,6\% nivel secundario.

El estado civil más recurrente es casado con un $42 \%$ y en unión libre con el $37,7 \%$ siendo unos porcentajes significativos en cuanto a los demás estados civiles. Los estratos socioeconómicos se encuentran distribuidos de la siguiente manera, estrato cero el $1,1 \%$, estrato 1 el $42,6 \%$, estrato 2 el $50,7 \%$ y estrato 3 el 5,3\%.

Los resultados de la escala de actitudes tipo Likert hacen referencia a las actitudes que tienen los conductores frente a las categorías de salud laboral, bienestar y percepción del trabajo, cada una de ellas contiene subcategorías que se muestran más adelante.

En la tabla 1, se presenta la información correspondiente a la primera categoría de la escala de actitudes, correspondiente a salud laboral (media de 2,26, deviación estándar de 0,301 y alfa de Cronbach's 0,758 ).

Tabla 1. Percepción de Salud laboral de conductores de transporte urbano

\begin{tabular}{lccc}
\hline & Frecuencia & \% & Media \\
\hline Muy favorable & 86 & 18,38 & \\
Favorable & 371 & 79,27 & 2,26 \\
Desfavorable & 11 & 2,35 & \\
Total & 468 & 100,0 & \\
\hline
\end{tabular}

Esta categoría a su vez contiene las subcategorías: condiciones de salud, que son complementados con los resultados del autorreporte de morbilidad sentida, y tres subcategorías más, condiciones de trabajo, estilos de vida y tiempo libre.

Los resultados de la tabla 2 muestran que los conductores presentan una actitud muy favorable respecto a compartir información de factores que intervienen sobre la salud; entre los factores más importantes para ellos son los hábitos alimenticios (73,5\%), higiene oral $(80,6 \%)$, beneficios de realizar actividad física $(75,6 \%)$, pausas activas en el trabajo $(76,7 \%)$, importancia de realizar actividad física $(86,3 \%)$, prevención de la hipertensión arterial $(76,9 \%)$, prevención de la diabetes $(80,1 \%)$, prevención de la obesidad (78\%) e información para la prevención de rinitis viral $(80,6 \%)$.

Igualmente la descripción de los datos más salientes sobre morbilidad sentida (antecedentes médicos y manifestaciones actuales) referentes al auto reporte de sus condiciones de salud, permite complementar la información en cuanto a la categoría de salud laboral y muestra que las molestias mayormente percibidas por los conductores son: dolores de espalda $(68,8 \%)$, cansancio visual $(56,2 \%)$, dolores de cintura $(50,2 \%)$, dolores de cabeza $(48,5 \%)$, dolores de cuello $(48,5 \%)$ y dolores de rodilla $(40,8 \%)$. Las manifestaciones actuales más percibidas son cansancio o aburrimiento con el 54\% y mencionan enfadarse con facilidad 37,6\%.

La tabla 2 igualmente muestra que el $59,6 \%$ de los conductores tiene una actitud desfavorable hacia las condiciones de trabajo, dentro de esta subcategoría los conductores consideran importante que, se realice una inspección de riesgos en el bus en que laboran $(88,5 \%)$, por otra parte, perciben que se encuentran expuestos a: contaminantes atmosféricos como el $\mathrm{CO}_{2}$ $(84,2 \%)$, vibraciones constantes en el lugar de trabajo $(65,2 \%)$, robos $(82,3 \%)$ y reconocen la importancia de conocer: las normas de tránsito para evitar accidentes en el vehículo (94,9\%), las normas de seguridad para evitar las enfermedades causadas por el trabajo $(93,6 \%)$, 
además perciben que las actividades que realiza en el trabajo son repetitivas $(93,2 \%)$ y que permanecen sentados largo tiempo en su trabajo $(93,4 \%)$.

Tabla 2. Subcategorías de Salud laboral de conductores de transporte urbano

\begin{tabular}{|c|c|c|c|c|}
\hline Subcategoría evaluada & Actitud & Frecuencia & $\%$ & Media \\
\hline \multirow{4}{*}{ Condiciones de salud } & Muy favorable & 379 & 81 & \multirow{4}{*}{1,26} \\
\hline & Favorable & 64 & 13,7 & \\
\hline & Desfavorable & 17 & 3,6 & \\
\hline & Muy desfavorable & 18 & 1,7 & \\
\hline \multirow{3}{*}{ Condiciones de trabajo } & Muy favorable & 7 & 1,5 & \multirow{3}{*}{2,58} \\
\hline & Favorable & 182 & 38,9 & \\
\hline & Desfavorable & 279 & 59,6 & \\
\hline \multirow{2}{*}{ Estilos de vida } & Muy favorable & 455 & 97,2 & \multirow{2}{*}{1,03} \\
\hline & Favorable & 13 & 2,8 & \\
\hline \multirow{4}{*}{ Tiempo libre } & Muy favorable & 84 & 17,9 & \multirow{4}{*}{1,94} \\
\hline & Favorable & 328 & 70,1 & \\
\hline & Desfavorable & 55 & 11,8 & \\
\hline & Muy desfavorable & 1 & 0,2 & \\
\hline
\end{tabular}

Dentro de los estilos de vida, que fue la tercera subcategoria evaluada, se encontró una actitud muy favorable $(97,2 \%)$ frente a los hábitos o comportamientos que determinan el estado de salud, los factores percibidos que inciden en la salud de los conductores son: el no contar con horarios estables para desayuno, almuerzo y cena $(53,6 \%)$, no consumir agua diariamente $(13,7 \%)$, consumir alimentos con altos porcentajes de grasas saturadas $(24,1 \%)$ y estresarse en el trabajo (42,9\%); por el contrario se puede tomar como un factor protector que el $44,2 \%$ de los conductores no consume comida snack (comida empaquetada), el $82,7 \%$ de los conductores consumen alimentos preparados en casa, a 81,2\% consideran importante comer de forma balanceada, al $82,1 \%$ les gusta practicar regularmente ejercicio físico, el $62,4 \%$ no fuma cigarrillo, el $96,8 \%$ no consumen ningún tipo de drogas psicoactivas, el $42,9 \%$ no consumen licor y el $91,2 \%$ consideran importante que en la empresa se promueva actividades recreativas, de esta manera la presente información es un insumo importante para la formulación de planes de mejoramiento en estilos de vida saludable para los conductores.

En lo correspondiente a tiempo libre, los conductores presentan una actitud favorable $(70,1 \%)$ frente a las actividades que son diferentes a las de su trabajo, dentro de estas las que realizan con mayor frecuencia son: ver televisión (58,1\%), salir de paseo $(36,3 \%)$, realizar deportes $(31,4 \%)$, dormir $(24,6 \%)$,ver películas $(43,6 \%)$ consumir licor en algunas ocasiones $(22,4 \%)$ y fumar cigarrillo algunas ocasiones $(12 \%)$, se puede observar que más de la mitad de los conductores optan por mirar televisión en su tiempo libre.

La segunda categoría de la escala de actitudes es la de bienestar, que se presenta en la tabla 3.

Tabla 3. Percepción de Bienestar de conductores de transporte urbano

\begin{tabular}{lrrr}
\hline & Frecuencia & \multicolumn{1}{l}{$\%$} & Media \\
\hline Muy favorable & 363 & 77,6 & \\
Favorable & 102 & 21,8 & 1,72 \\
Desfavorable & 3 &, 6 & \\
Total & 468 & 100,0 & \\
\hline
\end{tabular}

La categoría de Bienestar está compuesta por las sub categorías de bienestar laboral, familia y clima organizacional, las cuales se presentan en la tabla 4 .

En la subcategoría de Bienestar laboral se encontró que el $64,3 \%$ de los conductores presentan una actitud muy favorable sobre las 
relaciones laborales que tienen en su trabajo. De esta forma, los conductores consideran que se pueden comunicar de forma directa con: sus jefes $(64,3 \%)$, con sus compañeros $(74,8 \%)$ y el $74,1 \%$ manifiestan sentirse estables laboralmente, el $87,8 \%$ sentirse orgullosos de su oficio, el $74,4 \%$ afirman que pueden hacer uso de sus habilidades en el trabajo, el $78,8 \%$ afirman poder hacer usos de sus conocimientos y el $90,6 \%$ se sienten orgullosos de pertenecer al SETP.

Tabla 4. Subcategorías de Bienestar de conductores de transporte urbano

\begin{tabular}{|c|c|c|c|c|}
\hline Subcategoría evaluada & Actitud & Frecuencia & $\%$ & Media \\
\hline \multirow{4}{*}{ Bienestar laboral } & Muy favorable & 301 & 64,3 & \multirow{4}{*}{1,42} \\
\hline & Favorable & 140 & 29,9 & \\
\hline & Desfavorable & 26 & 5,6 & \\
\hline & Muy desfavorable & 1 & 0,2 & \\
\hline \multirow{3}{*}{ Familia } & Muy favorable & 416 & 88,9 & \multirow{3}{*}{1,11} \\
\hline & Favorable & 51 & 10,9 & \\
\hline & Desfavorable & 1 & 0,2 & \\
\hline \multirow{3}{*}{ Clima organizacional } & Muy favorable & 314 & 67,1 & \multirow{3}{*}{1,35} \\
\hline & Favorable & 146 & 31,2 & \\
\hline & Desfavorable & 8 & 1,7 & \\
\hline
\end{tabular}

En la subcategoría Familia los conductores presentan una actitud muy favorable hacia la convivencia con sus familias con un $88,9 \%$. Ellos manifiestan sentir: apoyo emocional por parte de sus familias ante cualquier situación $(91,5 \%)$, respaldo de su familia en las decisiones que toman (el 87\%), sienten que sus familias tienen confianza en ellos $(90,6 \%)$ y el $93,8 \%$ les gusta vivir con sus familias.

En los resultados referentes a Clima organizacional, los conductores manifiestan una actitud muy favorable frente a las percepciones respecto a la empresa. Consideran que sus compañeros de trabajo son: tolerantes (37\%), honestos $(41 \%)$, respetuosos $(43,6 \%)$, solidarios $(53,2 \%)$, asimismo expresan ser tolerantes con sus compañeros $(76,1 \%)$; por otra parte consideran que sus jefes inmediatos son respetuosos con ellos $(85,7 \%)$, y consideran importante que se oriente a las personas para que se sientan bien en su puesto de trabajo $(92,7 \%)$. En este sentido manifiestan que es importante poner en práctica dentro de la empresa valores como: el respeto $(94,9 \%)$ y la honestidad $(94,2 \%)$ de igual forma expresan que para fortalecer el clima organizacional se debe capacitar a los conductores en comunicación efectiva $(90,8 \%)$ y resolución de conflictos $(91,2 \%)$.

En cuanto a la categoría de Percepción del trabajo, los resultados generales de las actitudes se presentan en la tabla 5 y los resultados por las subcategorías, Satisfacción laboral y Atención al usuario se presentan en la tabla 6.

Tabla 5. Percepción del trabajo de conductores de transporte urbano

\begin{tabular}{lrrr}
\hline & Frecuencia & \% & media \\
\hline Muy favorable & 352 & 75,21 & \\
Favorable & 111 & 23,72 & 1,26 \\
Desfavorable & 5 & 1,068 & \\
Total & 468 & & \\
\hline
\end{tabular}

En este apartado el $74,1 \%$ de los conductores tienen una actitud favorable hacia la satisfacción laboral. La mayor parte de los conductores consideran que el trabajo ocupa una parte importante en sus vidas $(91,5 \%)$, se siente satisfechos con su trabajo $(86,1 \%)$ y sienten que las funciones que cumplen les gusta $(86,3 \%)$, no obstante el $81,2 \%$ considera que su trabajo los deja agotados. 
Tabla 6. Subcategorías de Percepción del trabajo de conductores de transporte urbano

\begin{tabular}{|c|c|c|c|c|}
\hline Subcategoría evaluada & Actitud & Frecuencia & $\%$ & Media \\
\hline \multirow{4}{*}{ Satisfacción laboral } & Muy favorable & 82 & 17,5 & \multirow{4}{*}{1,91} \\
\hline & Favorable & 347 & 74,1 & \\
\hline & Desfavorable & 37 & 7,9 & \\
\hline & Muy desfavorable & 2 & 0,4 & \\
\hline \multirow{3}{*}{ Atención al usuario } & Muy favorable & 453 & 96,8 & \multirow{3}{*}{1,03} \\
\hline & Favorable & 14 & 3,0 & \\
\hline & Desfavorable & 1 & 0,2 & \\
\hline
\end{tabular}

Media 1,88; desviación estándar 0,34; Alfa de Cronbach's 0,732

En esta última subcategoría, los conductores tienen una actitud muy favorable frente a recibir capacitaciones en atención al usuario (96,8\%), ya que consideran que: una mejor atención al usuario ayuda a atraer más usuarios $(93,4 \%)$ y que a través del saludo el usuario se siente más a gusto con el servicio (92,9\%).

\section{Discusión}

El objetivo de este estudio fue caracterizar la calidad de vida laboral percibida de los conductores desde la visión de la GTH en una muestra de 468 conductores, los resultados encontrados dan cuenta de la importancia de abordar y promover la salud laboral, el bienestar y la percepción del trabajo dentro del ambiente laboral, para mejorar la calidad de vida de todos los trabajadores. Esto es posible a través de la identificación de necesidades y la superación de cada una de ellas.

Los datos sociodemográficos muestran que el mayor porcentaje de los conductores del SETP está entre los 20 - 40 años, todos son hombres y han cursado la primaria y secundaria de manera incompleta $\left(2^{\circ}, 3^{\circ}, 4^{\circ}, 6^{\circ} \mathrm{y} 9^{\circ}\right)$, estos resultados son similares a otros estudios, el primero de ellos desarrollado en Colombia, en una muestra de 1100 historias clínicas laborales de la oficina de Salud Ocupacional de una Cooperativa de Transporte Público, donde se encontró que la población de conductores de transporte urbano era joven $(51 \%$ entre 23 y 33 años), mayoritariamente masculina y presentaba un bajo nivel de escolaridad $(46.5 \%$ sólo tiene primaria) ${ }^{30}$, en el segundo estudio desarrollado también en Colombia en una muestra de 194 conductores, se encontró una edad promedio de 36,7 años, la mayoría hombres que han alcanzado algún un nivel de escolaridad secundaria (el 52,1\%) ${ }^{31}$, y el tercero un estudio realizado en España en una muestra de 2005 conductores que a diferencia de los dos anteriores presenta un nivel de educación media en la mayoría de los casos y presencia de mujeres en el ejercicio de la conducción ${ }^{32}$; lo que denota que las empresas del sector transporte especialmente en Colombia deben interesarse en la formación académica de su personal ${ }^{30}$ donde el área de gestión del talento humano, a través de la identificación de capacidades, habilidades e intereses personales apoye en la formación del personal y su desarrollo de manera continua, potencializando fortalezas y brindándoles oportunidades de mejoramiento 33 .

Inicialmente, se encontró que los conductores tienen una actitud muy favorable frente a la salud laboral, expresando una disposición positiva para recibir información acerca de salud laboral, estilos de vida saludable, y actividades para realizar en el tiempo libre, que les permita a su vez manejar y controlar factores que inciden sobre su salud, lo que invita a fortalecer de manera permanente aspectos relacionados a su salud laboral a través de medios informativos, capacitaciones, entrenamientos, intervenciones, tratamientos, entre otros.

Al complementar los resultados referentes a salud laboral con la encuesta de morbilidad sentida, se encuentra en los conductores algunos malestares físicos (dolores de espalda, manos, cintura, cabeza, cuello y rodillas), malestares 
que según la revisión bibliográfica están relacionados con los estilos de vida, la postura inadecuada y el sedentarismo, este último es uno de los principales factores de riesgo para el desarrollo de diversos problemas de salud, principalmente de columna vertebral y por tanto dolores en la zona dorso lumbar ${ }^{34}$. Las variables identificadas con el autorreporte de morbilidad sentida son de importancia dentro de los sistemas de transporte debido a que estas pueden interferir con la salud mental y física del conductor además de la calidad de vida laboral.

La actitud muy favorable frente a la salud laboral en los conductores, pese a algunos malestares físicos reportados, se debe a que la mayoría de ellos no perciben como recurrentes dichos malestares, ya que el $60 \%$ son adultos en edades comprendidas entre los 20 y 40 años, que ingresaron recientemente a laborar dentro de la empresa, algunos van desde un día de labor hasta cinco años, y debido a la antigüedad en el cargo, es menor el tiempo de exposición a los riesgos laborales inherentes al cargo, por lo tanto no perciben o manifiestan afecciones de salud marcadas.

Ahora bien, la inactividad física (sedentarismo) y una dieta rica en grasas saturadas contribuyen con a la aparición de la obesidad, los resultados muestran que los conductores del SETP tienen una tendencia hacia el sobrepeso, ya que, el $54 \%$ sobrepasan los $80 \mathrm{~kg}$ y la estatura promedio 1,67. De forma similar se realizó un estudio en conductores mexicanos donde el $83,25 \%$ califican con sobrepeso, ${ }^{35}$ los resultados son más altos que los del presente estudio, pero igualmente en las dos investigaciones se analiza que los porcentajes son considerables y es necesario proponer programas para su abordaje. Sin embargo es fundamental resaltar la importancia que le dan los conductores del SETP a los beneficios de realizar actividad física con el $75,6 \%$, y aunque esta consideración no determina la conducta de hacer ejercicio, si predispone la ejecución conductual. En esta medida, se comprende que es indispensable el fortalecimiento del área de salud y, más aun, teniendo en cuenta que los conductores con problemas de salud son más propensos a tener algún tipo de accidente que los conductores saludables ${ }^{5}$.

Para favorecer el control de aquellos factores que afectan la salud y el bienestar laboral, el Ministerio del Trabajo de Colombia a través de la normatividad apoya la promoción de la salud y la prevención de enfermedades y accidentes laborales, específicamente por medio del Decreto 1443 de 201436, (que se encuentra dentro del Decreto 1072 de 2015) que tienen que ver con la implementación del Sistema de Gestión de Seguridad y Salud en el Trabajo (SGSST) 37 , en esta medida, la presente investigación es congruente con el objetivo de este decreto que busca la promoción de la seguridad y salud en el trabajo y la prevención de riesgos laborales, para evitar accidentes en el trabajo y enfermedades laborales. Al respecto de las condiciones de trabajo de los conductores se encontró una actitud desfavorable $(59,6 \%)$, puesto que identifican que se exponen a contaminantes atmosféricos como el $\mathrm{CO}_{2}(84,2 \%)$, vibraciones constantes en el lugar de trabajo (65,2\%), a robos (82,3\%), perciben que las actividades que realiza en el trabajo son repetitivas $(93,2 \%)$ y que permanecen sentados largo tiempo en su trabajo $(93,4 \%)$, información que permite conocer desde la perspectiva de los conductores los factores que inciden sobre su salud y posibilitando en un futuro la planeación de estrategias para minimizar sus efectos.

Por consiguiente, la seguridad y la salud en el trabajo al ser una disciplina que además de tratar la prevención de accidentes y enfermedades causadas por las condiciones de trabajo, y encargarse de la protección y promoción de la salud de todos los trabajadores, debe velar por la promoción y el mantenimiento del bienestar en todas las ocupaciones ${ }^{36}$.

Es por esto, que se indaga sobre las actitudes que tienen los conductores frente al bienestar laboral, donde se encuentra que poseen una actitud muy favorable hacia ella, pero, se mencionan algunas inconformidades respecto a las relaciones interpersonales con sus compañeros, lo que conlleva a reflexionar que, la mayor parte de los conductores considera que se 
deben afianzar los valores corporativos que influyen sobre el comportamiento organizacional, aspectos que de alguna manera se relacionan con la interacción entre compañeros; al respecto Bauman ${ }^{38}$ plantea que el debilitamiento de las relaciones interpersonales en este contexto se pueden relacionar a la existencia de intereses individuales y un alto nivel de desconfianza social, lo que conlleva como lo afirman Yañez, Arenas y Ripoll ${ }^{39}$, a una disminución de la cohesión grupal y desinterés colectivo, por esta razón, es conveniente tomar en cuenta estas variables puesto que existe una estrecha relación con los altos niveles de satisfacción laboral, esto hace que los trabajadores estén menos propensos al estrés, depresión y burnout ${ }^{40}$, que con frecuencia ocasiona ausentismo laboral.

Por otra parte, la influencia del apoyo familiar tiene efecto en la reducción de algunos estresores laborales, de esta manera aumenta la satisfacción y el bienestar de los trabajadores ${ }^{14}$, en este sentido, los resultados la información recolectada demuestran una concordancia con este planteamiento, debido a que los conductores manifiestan que sus familias les brindan apoyo emocional que es indispensable para ellos, y este se refleja a través de una actitud muy favorable frente a sus relaciones familiares, siendo un posible factor protector en el manejo de situaciones estresantes. La empresa entonces, debe considerar, no sólo enfocar su atención hacia el trabajador, sino incluir también a sus familias, puesto que fortalecer esta relación ayuda a vigorizar las redes de apoyo que posibilitan el desarrollo de mayor estabilidad emocional, compromiso económico y social para ellos y sus familias, obteniendo de esta relación beneficios tanto para el conductor como para la empresa.

Pese a las necesidades reflejadas en cuanto a relaciones interpersonales, la percepción que tienen los conductores del clima organizacional es muy favorable, sin embargo, Cárdenas et al. ${ }^{41}$, evidencian la importancia de tener en cuenta que los factores del clima organizacional pueden afectar procesos organizacionales y psicológicos, que influyen en la productividad, la satisfacción laboral y el bienestar laboral; por ello es indispensable proponer modelos que orienten la implementación de estrategias que fortalezcan el desarrollo humano y la percepción del trabajador.

Todo esto implica un cambio en la percepción del trabajador, entendido como un proceso cognitivo de la conciencia que consiste en el reconocimiento, interpretación y significación para la elaboración de juicios en torno a las sensaciones obtenidas del ambiente físico y social, del contexto laboral ${ }^{42}$ por medio de este proceso cognitivo el conductor pudo evaluar aquellas condiciones de su trabajo que ocasionan satisfacción en mayor o menor grado, en conjunto se pudo identificar una actitud favorable frente a su percepción laboral, dado que la mayor parte de la población expresaba sentirse satisfecha laboralmente, esto se debe a que los trabajadores consideran que la obtención de ingresos monetarios son parte esencial dentro del ámbito laboral. No obstante, afirman que sus labores y sus turnos laborales ocasionan a largo plazo un desgaste físico y emocional, que tiene una incidencia sobre las relaciones personales, la salud física y mental. La falta del establecimiento de un límite en horas de trabajo conduce al organismo a condiciones extremas que van más allá de la capacidad fisiológica del mismo para recuperase, estas largas jornadas laborales han mostrado invariablemente relación con el desempeño y eficacia en el trabajo, ocasionando errores en el cumplimiento de los sistemas de seguridad laboral aumentando síntomas de fatiga, disminución del estado de alerta, disminución de las horas de descanso y probablemente aumentando el riesgo de la aparición de síndromes metabólicos ${ }^{43}$.

Gracias a la evaluación de la percepción laboral se logró identificar inconformidades con el número de horas laborales, que según los conductores está afectando su estado de salud, entendido la salud no únicamente como ausencia de enfermedad como se había expuesto inicialmente, sino también como un estado de completo bienestar físico, mental y social ${ }^{19}$. En el nivel físico se manifiestan la aparición de fatiga y los dolores identificados por medio del 
autorreporte de morbilidad sentida, a nivel mental se encuentra el estrés y la irritabilidad y en el nivel social el trabajo en solitario y disposición del poco tiempo para el contacto social, además, manifiestan de manera general la necesidad de modificar puestos de trabajo y horarios laborales que les permita disponer de tiempo libre para la realización de actividades deportivas, recreativas y de formación académica.

De ahí, que la percepción laboral juega un papel importante en la valoración de la satisfacción laboral, Eljaiek ${ }^{44}$ menciona que la evaluación de la satisfacción laboral se hace necesaria, ya que un conductor satisfecho muestra mejor actitud que el que no, y este a su vez, exterioriza esa satisfacción mostrándose alegre y con disposición para el trabajo: y para el caso de los conductores, evaluados a pesar de mostrar una disposición positiva a ofrecer un buen servicio como lo muestran los resultados (actitud muy favorable hacia la atención al usuario con $96,8 \%$ ), en términos prácticos generalmente no lo hacen, ya que los factores multicausales siguen afectando la prestación de un buen servicio y desemboca en una atención al usuario poco apropiada. Zamora y $\mathrm{Cruz}^{23}$ al igual que Eljaiek ${ }^{44}$ resaltan la importancia de la satisfacción laboral, pero al mismo tiempo incorporan otros elementos como la valoración positiva del trabajo, el apoyo social y el control sobre el trabajo como factores protectores contra enfermedades físicas y trastornos mentales ${ }^{23}$.

En torno a lo planteado, la visión de la gestión humana debe integrar a la luz de los resultados, programas enfocados la superación de las necesidades expresadas por los conductores en busca del desarrollo y la gestión de cambio ${ }^{44}$ a través de la consolidación de la gestión del talento humano desde su comprensión e institucionalización como función estratégica de la empresa, en procura del mejoramiento de la calidad de vida y la productividad, a través promoción de la salud laboral, el bienestar laboral, la percepción laboral y la reducción de enfermedades y accidentes laborales. De este modo se puede apuntar de manera holista a los objetivos organizacionales mediante el apoyo a las estrategias empresariales y el desarrollo de las personas ${ }^{45}$.

Para lograr el desarrollo de estos programas, Lozano $^{33}$ menciona que es importante tener en cuenta las variables psicológicas (motivación, inteligencia, percepción, atención, emociones, lenguaje, entre otros) que interactúan con las variables contextuales y determinan la conducta organizacional $^{33}$; Castillo ${ }^{46}$ por su parte afirma que es conveniente empezar por identificar las necesidades y luego jerarquizarlas, para orientar específicamente los objetivos, políticas, normas y procedimientos del nuevo programa, y para que dichos programas de intervención sean aceptados con mayor facilidad, es necesario tener en cuenta los aportes proporcionados por los trabajadores ${ }^{45}$.

Retomando lo anteriormente expuesto, se puede decir que se abordó los objetivos planteados, sin embargo, surgen interrogantes en torno a ¿Cómo modificar los estilos de vida y las conductas no saludables, que promuevan la salud? ¿Cómo se debe impulsar el manejo de pausas activas en el trabajo? y ¿Cómo utilizar los factores protectores como la familia como un potencial motivador en el trabajo?, dado que estos pueden tener una fuerte conexión con la salud laboral.

\section{Conclusiones y recomendaciones}

La investigación requiere efectuar un trabajo interdisciplinario puesto que la calidad de vida es una variable transversal a todas las categorías abordadas y esta necesita de los aportes de diferentes disciplinas, como la psicología, la medicina, la terapia ocupacional e ingeniería industrial, entre otras, las cuales pueden garantizar el fortalecimiento de la calidad de vida a través de la promoción de la salud y prevención de enfermedades y accidentes laborales. Lo que da cuenta de la necesidad de un Sistema de Gestión de Seguridad y Salud en el Trabajo (SGSST) que responda a las necesidades de los colaboradores y que garantice el buen funcionamiento y control del estado de salud de sus trabajadores a través de exámenes pre ocupacionales, periódicos y post ocupacionales. 
A través del artículo se evidencia un aporte significativo en el SETP, en primer lugar al permitir la caracterización de la calidad de vida laboral desde el punto de vista de la gestión humana a partir de la percepción de los conductores que laboran en la empresa, conociendo las características sociodemográficas para la implementación de políticas y estrategias, como también la identificación, descripción y análisis de las necesidades que los trabajadores consideran primordiales según su percepción y sentir en las categorías y subcategorías abordadas.

En cuanto a salud laboral se logró identificar actitudes para la recepción de información relevante que posibilite la prevención de enfermedades laborales, acciones o hábitos en beneficios o no de la salud, reconocimiento de implicaciones propias del trabajo y de las condiciones de trabajo como la exposición a riesgos laborales, la importancia de conocer normas de tránsito y normas de seguridad para evitar accidentes de tránsito que para el caso de la población también son laborales, además de evitar enfermedades causadas por el trabajo, lo anterior teniendo en cuenta que un problema de salud interfiere en todas las dimensiones de la vida del trabajador.

En segundo lugar, permitió la comprensión respecto a las categorías de trabajo que están interconectadas, es decir que la afectación de una de ellas, sea salud laboral, bienestar laboral o percepción del trabajo se ve reflejada en las demás.

En tercer lugar, para que el SETP fortalezca la atención al usuario es importante continuar desarrollando la política de gestión del talento humano, como por ejemplo a través de la identificación de factores de riesgo y protectores en salud laboral en su capital humano.

En cuarto lugar, el presente estudio permitió establecer un punto de inicio para el desarrollo de líneas de investigación y profundización en las categorías de salud laboral, bienestar laboral y percepción laboral, cuya valoración fue realizada de forma global, en relación a esto, se sugiere indagar de forma específica en cada una de estas categorías para conocer un panorama detallado del entorno laboral de los trabajadores, contribuyendo de esta manera a una caracterización de la población cada vez más detallada.

Para finalizar, fomentar la promoción y mejoramiento de la salud laboral, proporciona beneficios significativos para la empresa y sus conductores, puesto que estas actividades, influyen tanto en la percepción que se tiene del ambiente laboral y el bienestar, como también en el estado de la salud física, y en un sentido opuesto, al afectarse esta categoría podría ocasionar una disminución de la calidad de vida y una reducción de la productividad laboral.

Se sugiere abordar en próximas investigaciones con mayor profundidad las categorías de condiciones de salud, condiciones de trabajo, estilos de vida, tiempo libre, relaciones interpersonales, bienestar, clima organizacional, percepción laboral y atención al usuario que permitan fortalecer líneas de intervención.

\section{Agradecimientos}

Se agradece al Programa de Psicología de la Universidad de Nariño por el conocimiento y apoyo para la realización de la presente investigación, a la Unión Temporal Ciudad Sorpresa por el apoyo logístico, por su interés y por hacer posible este proceso de práctica profesional, a ARL Positiva por sus asesorías, a todos los conductores que participaron en la investigación y a los estudiantes del Programa de Psicología matriculados a séptimo y primer semestre del periodo A de 2015 por el apoyo en la recolección de información.

Conflicto de intereses: Ninguno declarado por los autores.

\section{Referencias}

1. Rojas F, Mello C. El transporte público colectivo en Curitiba y Bogotá. Revista de Ingeniería. 2005;21:106$115 . \quad$ Disponible en: http://www.redalyc.org/articulo.oa?id=12101421801 0 
2. Vásquez, Acevedo y Blasa. Las TIC'S en el sistema de transporte urbano y el cambio organizacional en las empresas operadoras 2012. Administración y Organizaciones, 2012;15:139-152. Disponible en: http://web.a.ebscohost.com/ehost/detail/detail?sid=0 0c55e31-ae83-4f1a-bda9-

bedef23265ef\%40sessionmgr4004\&vid=0\&hid $=4112 \&$ bdata=Jmxhbmc9ZXMmc2l0ZT1laG9zdC1saXZl\#db=zb $\mathrm{h} \& \mathrm{AN}=85127919$

3. Betanzo E, Romero J, Obregón S. Un referencial para evaluar la gestión pública en transporte urbano de carga. Gestión y Política Pública, 2013;2:313-354. Disponible

en: http://web.a.ebscohost.com/ehost/pdfviewer/pdfview er?sid=b0160709-c2c8-48a7-9a95-

7 da789bcd08b\%40sessionmgr4004\&vid=1\&hid=4112

4. Moyano E, Mladinic A. Exposición, percepción de peligrosidad y de control de riesgos y comportamiento vial en peatones y conductores. Psykhe. 2011;10:135145.

5. Guache H, Suárez T, Gutiérrez F, Martínez C. Estado de salud en conductores profesionales que circulan por la vía Blanca. Revista cubana de Salud Pública. 2006;32: 126-130.

6. Rey J, Rosales E. Cansancio y somnolencia durante el desempeño laboral de los conductores interprovinciales: experiencia peruana y planteamiento de propuestas. Revista Peruana de Medicina Experimental y Salud Pública. 2010;27:237-42.

7. Ministerio de Transporte. Transporte en cifras, estadísticas. Colombia, 2014. Disponible en: https://www.mintransporte.gov.co/Documentos/docu mentos_del_ministerio/Estadisticas

8. Congreso de Colombia. Ley 1562 de 2012. Disponible en

http://wsp.presidencia.gov.co/Normativa/Leyes/Docu ments/ley156211072012.pdf

9. Ministerio de Trabajo. Enfermedades y accidentes laborales en cifras, 2013. Disponible en: http://www.mintrabajo.gov.co/medios-junio-

2012/1995-nuevas-condiciones-para-trabajar-en-lasalturas.pdf

10. Fasecolda. Enfermedad laboral en Colombia. 2013. Disponible en: http://ccs.org.co/img/Enfermedad\%20Laboral\%20en \%20Colombia\%20Fasecolda.pdf

11. Szu-Chia Chen, Jer-Ming Chang, Ming-Yen Lin, MengLing Hou, Jer-Chia Tsai, Shang-Jyh Hwang, Hung-Chun Chen. Association of Metabolic Syndrome and Albuminuria with Cardiovascular Risk in Occupational Drivers. International Journal of Molecular Sciences. 2013; 14: 21997-22010.

12. Naranjo M. Una revisión teórica sobre el estrés y algunos aspectos relevantes de éste en el ámbito educativo. Revista Educación. 2009;33:171-190. Disponible en: http://revistas.ucr.ac.cr/index.php/educacion/article/ view/511

13. Pacheco-Tabuenca T. Apoyo psicosocial a los pacientes en situación de emergencia. Ed. Arán, 2009:60-66. Disponible https: //books.google.com.co/books?id=2RYdca_w8dYC $\& p g=P T 28 \& d q=e s t r e s+$ positivo+y+negativo\&hl=es\&sa =X\&ved=0CDIQ6AEwBWoVChMI3K-

14. Córdoba $C$, Moreno $M$. Instrumento para evaluar variables psicológicas y laborales en conductores de autobuses. Reflexiones. 2013;92:109-122. Disponible en: http://www.redalyc.org/pdf/729/72927462008.pdf

15. Boada J, Prizmic A, González S, Vigil A. Estresores laborales en conductores de autobuses (ElBus-21). Universitas Psychologica, 2013;12:249-259. Disponible en:

http://revistas.javeriana.edu.co/index.php/revPsycho/ article/view/1825

16. Rocha A, Moraes D, Vitta Ad, Padovani CR, Plácido da Silva JC. Postura sentada: a eficácia de um programa de educação para escolares. Ciência \& Saúde Coletiva. 2004: 191-199. Disponible en: http://oai.redalyc.org/articulo.oa?id=6301349901 9.

17. Farmer J. Driven to it. Occupational Health. 2010; 62: 22-24. Disponible en: http://web.b.ebscohost.com/ehost/detail/detail?sid=b bd4a8af-3ffb-4167-8a5b-

a1c96fc9ab4a\%40sessionmgr114\&vid=1\&hid=115\&bd ata =Jmxhbmc9ZXMmc210ZT1laG9zdC1saXZl\#AN=5130 3509\&db=aph

18. Dos Santos E, Marquez R, Cardoso T, Leal C. Fatores de risco para doença arterial coronária em motorista de ônibus. Revista Baiana de Enfermagem, 2014; 28: 252259.

19. Organización Mundial de la Salud. Salud de los trabajadores: Plan de acción mundial. 2007. Disponible en:

http://www.who.int/occupational_health/WHO_health _assembly_sp_web.pdf?ua $=1$

20. Caracterización del ausentismo laboral en un centro médico de I nivel. Universidad del Rosario. Trabajo de grado para optar al título de Especialista en Salud Ocupacional. 2001. Disponible en: http://repository.urosario.edu.co/bitstream/handle/1 0336/2834/22647541-

2012.pdf; jsessionid=45DDBD4B293BF6B56317A7CD0 6D47262?sequence $=1$

21. Ardila R. Psicología, de la biología a la cultura. Coordinación de Publicaciones F.C.H. 2003. Disponible en http://www.bdigital.unal.edu.co/1314/2/01PREL01.p df

22. Marín M, Pico M. Conceptos de salud ocupacional. fundamentos en salud ocupacional. Colombia: Editorial Universidad de Caldas, Ciencias para la Salud; 2004: 16-18. Disponible https://books.google.com.co/books?id

23. Zamora M, Cruz M. La importancia de la valoración del trabajo en un grupo de conductores de carga. Enseñanza e investigación en psicología. 2012; 17: 209 $220 . \quad$ Disponible en: http://www.cneip.org/documentos/13.pdf

24. Bolaños G. Educación por medio del movimiento y la expresión corporal. Costa Rica: EUNED, 1991. 
Disponible

en:

https://books.google.com.pe/books?id=IvisGiBiafoC\&p rintsec=frontcover\&hl=es\&source=gbs_ge_summary_r $\& \mathrm{cad}=0 \# \mathrm{v}=$ onepage $\& \mathrm{q} \& \mathrm{f}=$ false

25. Llaneza A. Clima laboral. Ergonomía y psicología aplicada: Manual para la formación del especialista. España: Lex Nova; 2007: 466. Disponible en https://books.google.com.co/books?id=o6kLIwAFTvA C\&pg=PA466\&dq=percepcion+en+el+trabajo\&hl=es\&s

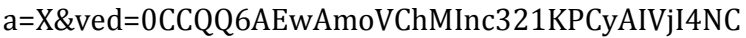
$\mathrm{h} 2 \mathrm{SJgPD} \# \mathrm{v}=$ onepage $\& \mathrm{q}=$ percepcion $\% 20 \& \mathrm{f}=$ false

26. Hinestroza F, Giraldo J. Riesgo Cardiovascular en conductores de servicio público intermunicipal. Revista Médica de Risaralda. 2008;15(2).

27. Giraldo J. Manual para los seminarios de investigación en psicología: profundización conceptual y textual. Colombia: Universidad Cooperativa de Colombia. 2006: 56. Disponible

en: https://books.google.com.co/books?id=9QxB0XqtFuUC $\& p g=P A 56 \& d q=e n f o q u e+$ cuantitativo\&hl=es\&sa=X\&ei =AofsVIqfGo7zggT03oD4BQ\&ved=0CEMQ6AEwBw\#v= onepage \&q=enfoque $\% 20$ cuantitativo $\& \mathrm{f}=$ false

28. Bernal C. Investigación descriptiva. Metodología de Investigación: Para administración, economía, humanidades y ciencias sociales. México: Pearson. 2006: $113 . \quad$ Disponible en: https://books.google.com.co/books?id=h4X_eFai59oC \&printsec $=$ frontcover $\& \mathrm{hl}=\mathrm{es} \# \mathrm{v}=$ onepage $\& \mathrm{q} \& \mathrm{f}=$ false

29. Colombia. Ministerio de la Protección Social. Ley 1090 de 2006, Por la cual se reglamenta el ejercicio de la profesión de la psicología, se dicta el código deontológico y bioético y otras disposiciones. Bogotá DC: Ministerio; 2006. Disponible en: http://www.upb.edu.co/pls/portal/docs/PAGE/GPV2_ UPB_MEDELLIN/PGV2_M030_PREGRADOS/PGV2_M03 0040020_PSICOLOGIA/CODIGO_ETICO/CODIGO\%20D EONTOLOGICO\%20Y\%20BIOETICO.PDF

30. Arbeláez L, Delgado C, Giraldo S, Gutiérrez C, Muñoz L. Características clínicas y patológicas de conductores de una empresa de transporte público 2003. Investigaciones Andina. 2004;8:14-21. Disponible en: www.redalyc.org/articulo.oa?id=239017799003

31. Chaparro E, Guerrero J. Condiciones de trabajo y salud en conductores de una empresa de transporte público urbano. Rev. Salud Pública. 2001 Junio 3(2):171-187. Disponible

en: http://www.scielo.org.co/scielo.php?script=sci_arttext \&pid=S0124-00642001000200005\&lng=en

32. Martos J, Roa J, Tortosa, L. Los conductores reexaminados: El transcurso del tiempo deteriora significativamente los conocimientos de los conductores. Revista Latinoamericana de Psicología 2012;44(3):83-94. Disponible en: http://www.scielo.org.co/scielo.php?script=sci_arttext \&pid=S0120-05342012000300007\&lng=en\&tlng=es.

33. Lozano L. El talento humano, una estrategia de éxito en las empresas culturales. Revista Escuela de Administración de Negocios. 2007;60:147-164. Disponible en

http://www.redalyc.org/pdf/206/20606008.pdf
34. Castillo J, Cubillos A, Orozco A y Valencia J. El análisis ergonómico y las lesiones de espalda en sistemas de producción flexible. Revista ciencias de la salud. 2007;5:43-57. Disponible en: http://www.redalyc.org/pdf/562/56250305.pdf

35. González M, Landero R, Maruris M, Cortés P, Vega R y Godínez F. Estrés cotidiano en trabajadores al volante. Pepsic. 2013;10. Disponible en: http://pepsic.bvsalud.org/scielo.php?pid=S0719448x2013000100008\&script=sci_arttext

36. Colombia, Ministerio del Trabajo. Decreto 1443 de 2014. Disposiciones para la implementación del sistema de gestión de la seguridad y salud en el trabajo. Bogotá DC: Ministerio; 2014. Disponible en: http://www.mintrabajo.gov.co/normatividad-juliodecretos-2014/3700-decreto-1443-del-31-de-julio-de2014.html

37. Colombia, Ministerio del trabajo. Decreto 1072 de 2015. Por el cual se expide el decreto único reglamentario del sector trabajo. Bogotá DC: Ministerio; 2015. Disponible en: http://unidad.serviciodeempleo.gov.co/documentos/D ecreto1072-2015.pdf

38. Bauman Z. La Sociedad sitiada. México: Fondo de Cultura Económica. 2007.

39. Yañez, R, Arenas M, Ripoll M. El Impacto de las relaciones interpersonales en la satisfacción laboral general Liberabit. Revista de Psicología. 2010;16:193$201 . \quad$ Disponible en: http://www.redalyc.org/articulo.oa?id=68617161008

40. Faragher E, Cass M, Cooper C. The relationship between job satisfaction and health: a meta-analysis. Occupational and Environmental Medicine. 2005;62,105-112.

41. Cárdenas L, Arciniegas Y, Barrera M. Modelo de intervención en clima organizacional International. Journal of Psychological Research, 2009;2:121-127. Disponible en: http://www.redalyc.org/articulo.oa?id=29902351300 5

42. Sepúlveda. Bienestar psicológico laboral en las organizaciones empresariales de la ciudad de Medellín, Colombia. 2013.

43. Juárez A, Sánchez R, Haro L, Larios E, Justificaciones médicas de la jornada laboral máxima de ocho horas. Revista Médica del Instituto Mexicano del Seguro Social. 2007;45:191-197. Disponible en: http://www.redalyc.org/articulo.oa?id=457745526 014. Fecha de consulta: 14 de noviembre de 2016.

44. Eljaiek G. Sistema nuevo, conductores nuevos (se presume). Universitas Psychologica. 2005;4:307-315. Disponible http://www.scielo.org.co/scielo.php?script=sci_arttext \&pid=S1657-92672005000300005

45. Calderón H, Murillo S, Torres K. Cultura organizacional y bienestar laboral. Cuadernos de Administración. 2003; 16: 109-137. Disponible en: http://www.redalyc.org/pdf/205/20502506.pdf

46. Castillo A. Definición e importancia del bienestar laboral. Administración de personal: un enfoque hacia la calidad. Colombia: Ecoes ediciones. 2010. 